\title{
High power cladding-pumped Raman fiber laser with true single-mode output at $1660 \mathrm{~nm}$
}

\author{
C. A. Codemard, P. Dupriez, Y. Jeong, J. K. Sahu, M. Ibsen, J. Nilsson \\ Optoelectronics Research Centre, University of Southampton, Southampton SO17 1BJ, England \\ Phone: +44 (0) 2380593143 , fax: +44 (0)238059 3142 \\ Email: cac@orc.soton.ac.uk
}

\begin{abstract}
We report a $10 \mathrm{~W}$ continuous-wave cladding-pump Raman fiber laser with single-mode output at $1660 \mathrm{~nm}$ pumped by a high-power Er:Yb fiber amplifier. The laser threshold was $6.5 \mathrm{~W}$ and its slope efficiency was $67 \%$.
\end{abstract}

OCIS codes: (140.3550) Lasers, Raman, (140.3510) Lasers, fiber

\section{Introduction}

High-power fiber laser operating in the eye-safe wavelength regime around $\sim 1.5-2 \mu \mathrm{m}$ with diffraction limited output beam have numerous applications. Recently, there has been dramatic progress in scaling the output power from cladding-pumped Er: $\mathrm{Yb}[1,2]$ and $\mathrm{Tm}$ [3] doped silica fiber lasers operating in the $1.53 \mu \mathrm{m}-1.62 \mu \mathrm{m}$ and 1.85 $-2.1 \mu \mathrm{m}$ wavelength range respectively. However, these fiber lasers cannot operate between $1.62 \mu \mathrm{m}$ and $1.85 \mu \mathrm{m}$ due to the absence of efficient radiative transition in rare-earth ions at these wavelengths. Instead, Raman fiber lasers can be used to reach this wavelength range. Raman fiber laser can convert effectively light at any short wavelength into a longer wavelength within a broad bandwidth at the Stokes frequency downshift. Nevertheless, a conventional Raman fiber laser requires a transverse single-mode pump source which even when fiber lasers are used is difficult to achieve at high powers. In particular, high-power Raman lasers in the eye-safe range are normally pumped with Er: $\mathrm{Yb}$ co-doped fiber lasers, which, however, because of a typically large numerical aperture are difficult to make single-moded. An alternative to the traditional core-pumped Raman fiber laser is to use a multimode fiber as the Raman gain medium and, for single-mode operation, select only the fundamental mode to lase. Such devices can also be seen as brightness converters that rely on the capability for beam "clean-up" of stimulated Raman scattering [4]. For example, in [5] a fiber Bragg grating (FBG) written in a multimode fiber did allow a multimode Raman fiber laser to achieve a good beam quality with a $\mathrm{M}^{2}$ of 1.66. Still the output power was limited to less than $1 \mathrm{~W}$ and the fiber output was not readily compatible with a single-mode fiber, which is often a requirement. On the other hand, based on a similar principle, we demonstrated earlier a $3.4 \mathrm{~W}$ output Raman fiber laser [6]. In this case, a double-clad fiber with a raised index core which supports only the fundamental mode of the desired operating wavelength was used. Compared to [5], our solution leads to a smaller fundamental mode which can be a drawback because of damage and also because the overlap with some of the pump modes can be small. However, appropriately designed inner claddings (e.g., with a D-shape) or mixing of the pump modes mitigates the overlap issue, and for many devices damage is not a concern. Furthermore the double-clad structure helps in mode-selection and suppression of mode-coupling at the Stokes because of the greater difference in shape and effective index between the fundamental mode and higher-order modes. Thus mode selection can be achieved by a FBG with pitch adjusted for the effective index of the fundamental core-mode. Furthermore, the output of the double-clad fiber is matched to standard single-mode fiber which can be used to further increase the modal discrimination.

We here present an improved double-clad Raman laser which, compared to our previous work [6], provides a higher output power (>10 W at $1660 \mathrm{~nm}$ ) and an improved degree of brightness enhancement, from $\mathrm{M}^{2}=\sim 8.5$ to a diffraction-limited beam. We use a new cavity design with an output port made of standard single-mode fiber (SMF28). This makes the fiber laser truly single-mode and fully compatible with such fiber.

\section{Experimental set-up}

The experimental set-up consists of a high-power continuous-wave (cw) pump source at $1552 \mathrm{~nm}$ and of an all-fiber cladding-pumped Raman fiber laser as shown in fig. 1. The pump source is a two-stage fiber master-oscillator power-amplifier (MOPA) based on Er:Yb co-doped fiber amplifiers (EYDFAs). The MOPA's seed source consists of a high-power tunable fiber ring laser which uses a commercial 2 W EYDFA from Southampton Photonics and a tunable fiber Bragg grating made in-house (e.g. [7]). The ring laser was set at $1.4 \mathrm{~W}$ of output power at $1552 \mathrm{~nm}$. The ring laser was designed so that its linewidth of $0.2 \mathrm{~nm}$ was large enough to avoid stimulated Brillouin scattering 
in the relatively long Raman fiber laser. The seed source was then free-space coupled through a pair of lenses and a dichroic mirror into a $4 \mathrm{~m}$ long large-core high-power EYDFA. The erbium-ytterbium doped fiber had a core diameter of $30 \mu \mathrm{m}$ and a NA of 0.2 . The inner-cladding was D-shaped with size optimized for good pump coupling. The diameter was $650 \mu \mathrm{m}$ and the NA (i.e., for the pump waveguide) was 0.48 , as determined by a low-index polymer coating. Both end-facets of the large-core EYDF were angle-polished to suppress back-reflections. In this experiment, we used a diode stack pump source at $972 \mathrm{~nm}$ capable of delivering up to $1 \mathrm{~kW}$ of cw power to pump the high-power EYDFA. An output power in excess of $35 \mathrm{~W}$ was obtained from the MOPA as shown in fig. 2 with a clean spectrum (fig. 2 inset). The slope efficiency of the high-power EYDFA was somewhat low, $21 \%$, because the EYDF length was not optimized for the $1552 \mathrm{~nm}$ wavelength required in his experiment. The output beam quality of the MOPA corresponds to a $\mathrm{M}^{2}$ of $~ 8.5$. Even for our large core, the beam quality is worse than normal (e.g. [2]), but for this application a single-mode pump source is not required. The $972 \mathrm{~nm}$ pump is launched with the $1552 \mathrm{~nm}$ signal into the EYDFA so that the fiber output end can be free-spaced launched into the double-clad Raman fiber (DCRF) laser. It would also allow the output end of the EYDF to be directly spliced to the DCRF, but because of the core mismatch this was not attempted. The remaining unabsorbed pump power at $972 \mathrm{~nm}$ is removed by a dichroic mirror. The signal is then further reflected in dichroic mirror which transmits wavelengths longer than $1650 \mathrm{~nm}$ and is then launched into the DCRF inner-cladding via a lens. The launch efficiency was $60 \%$ into the DCRF.

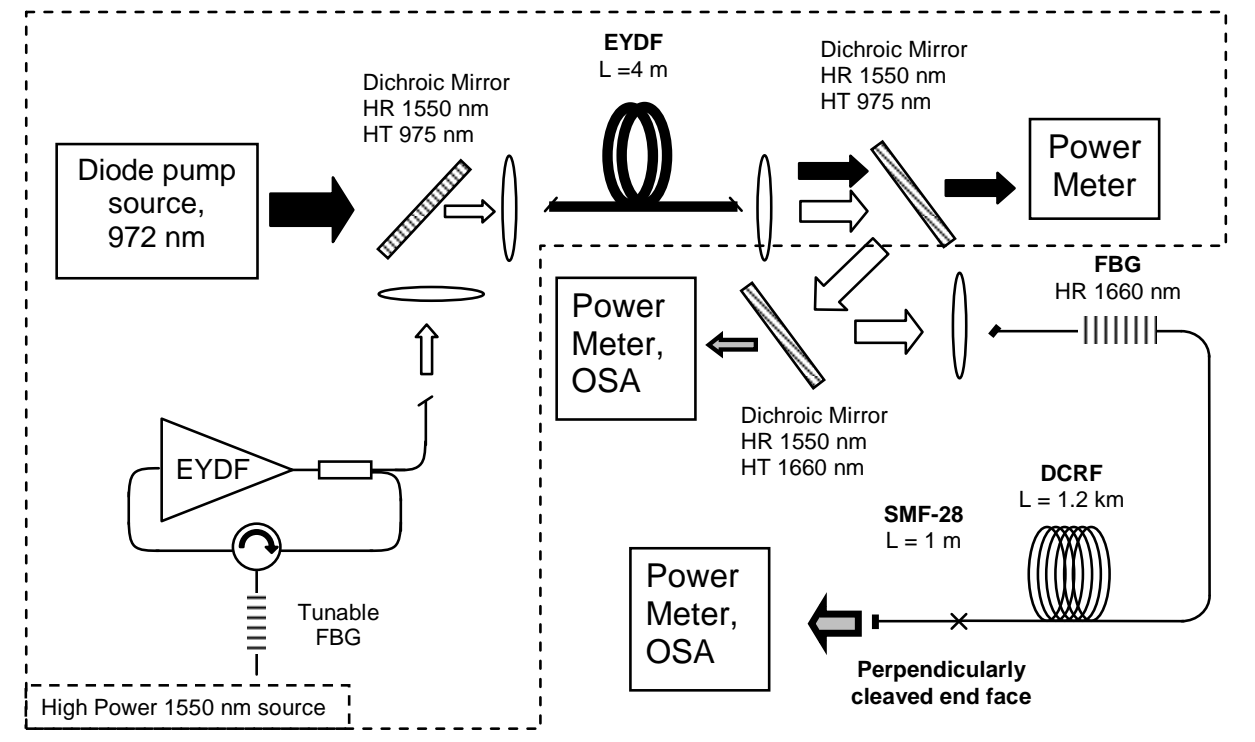

Figure 1 : Experimental set-up

The Raman gain medium is a special double-clad fiber manufactured in-house using a standard MCVD process. The DCRF has a pure silica outer cladding. The inner cladding and core are germanosilicate with different germanium contents to enhance the Raman process and to define the desired waveguide structure. The diameter of the inner cladding is $21.6 \mu \mathrm{m}$ and its NA is 0.22 with respect to the outer cladding. The core diameter is $9 \mu \mathrm{m}$ and its NA is 0.14 with respect to the inner cladding. The core has an estimated cut-off wavelength of $1630 \mathrm{~nm}$. The core and inner cladding propagation loss at $1552 \mathrm{~nm}$ are $3.1 \mathrm{~dB} / \mathrm{km}$ and $2.1 \mathrm{~dB} / \mathrm{km}$, respectively. The fiber in our laser was $1.2 \mathrm{~km}$ long and its output end is spliced to a 1 meter long piece of SMF-28. The typical splice loss for the core mode is less than $0.5 \mathrm{~dB}$ while all other modes are lost at this point to un-guided radiation modes. We used a highindex gel to strip all the unwanted modes and also to protect the high-index coating of the SMF fiber.

The Raman laser cavity itself consists of a narrowband $(\sim 0.4 \mathrm{~nm})$ highly-reflective FBG at $1660 \mathrm{~nm}$ written in the core of the DCRF at the $1552 \mathrm{~nm}$ launch end with the laser output coupler formed by a flat cleave in the SMF- 28 fiber. Thus the laser output is a true single-mode beam and can readily be spliced to conventional telecomcompatible fiber, if, e.g., a low-reflecting FBG is used as an output coupler. The HR FBG has a reflection of $80 \%$. Thus we expect to have some leakage from the Raman laser in the backward direction. Hence we used a protective dichroic mirror to prevent the $1660 \mathrm{~nm}$ radiation from being fed back into the MOPA.

\section{Results}

The output power from Raman laser through the SMF-28 output end is shown in fig. 3. The laser threshold is around $6.5 \mathrm{~W}$ of launched pump power at $1552 \mathrm{~nm}$. This is similar to our earlier work [6]. The slope efficiency of the laser is $67 \%$ and the conversion efficiency close to $47.6 \%$ at the maximum power. A maximum output power of $10.2 \mathrm{~W}$ 
was obtained for $21.4 \mathrm{~W}$ of launched pump power. The limited reflectivity of the FBG in the pump launch end led to a non-negligible amount of output power lost at this point. Including this lost power, the total slope efficiency of the laser is in fact $84 \%$ with a conversion efficiency of $60 \%$ as shown in figure 3 . Hence the laser performance could be further improved with a better FBG, as well as with a fiber with a lower background loss. Still even with the present parameters the DCRF is highly efficient, and considering the background loss, the double-ended slope efficiency even exceeds the quantum limit. A reason for this could be the nonlinear absorption characteristics of a Raman laser, which can lead to slope efficiencies above the quantum limit over a limited range. However, taking the background loss into account, also the total conversion efficiency is very close to the quantum limit, or even exceeds it. This would be unphysical but could be explained by an under-estimated pump launch.

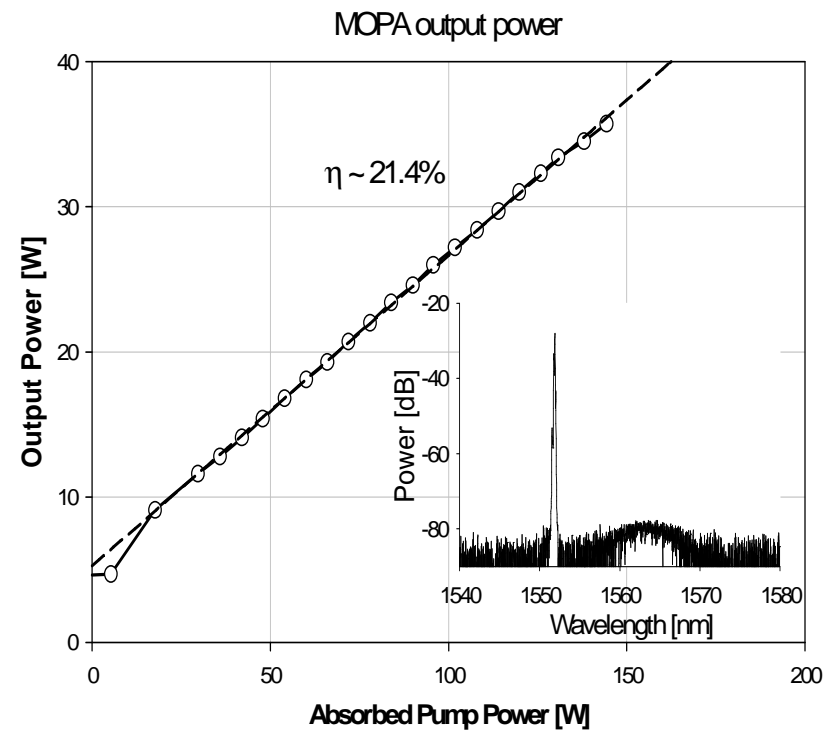

Figure 2 : MOPA output power at $1552 \mathrm{~nm}$, inset: output spectrum with a resolution of $0.05 \mathrm{~nm}$

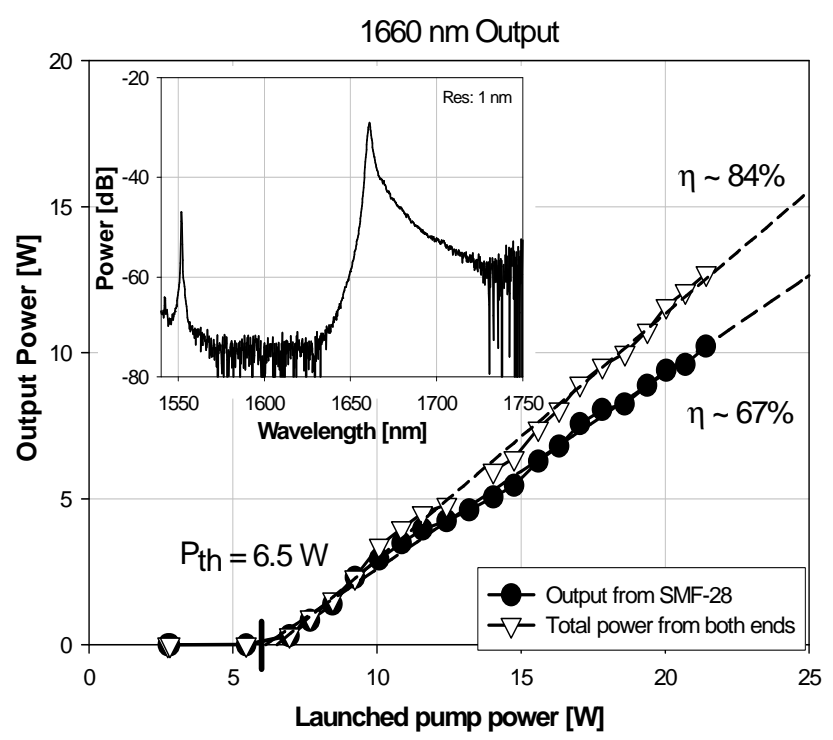

Figure 3: Cladding-pump Raman laser at $1660 \mathrm{~nm}$ characteristics: output powers and spectrum at the maximum output power (inset)

The output spectrum measured at the maximum output power with a resolution of $1 \mathrm{~nm}$ is shown in the inset of fig. 3. The remaining pump power in the single-mode core of the SMF is $20 \mathrm{~dB}$ lower than the laser output at $1660 \mathrm{~nm}$. Furthermore a broadening of Raman fiber laser linewidth (FWHM) from $0.5 \mathrm{~nm}$ (near the threshold) to $2.5 \mathrm{~nm}$ (at the maximum output) is observed. This is caused by the combination of the high intensity in the core mode of the lasing wavelength and four-wave mixing in the Raman fiber laser [8].

\section{Summary}

In conclusion, we have demonstrated an efficient high-power $\mathrm{cw}$ single-mode cladding-pumped Raman fiber laser at $1660 \mathrm{~nm}$ based on a double-clad germanosilicate fiber. The Raman fiber laser yielded $10.2 \mathrm{~W}$ of single-ended output power for $21.4 \mathrm{~W}$ of pump power at $1552 \mathrm{~nm}$. The laser threshold was $6.5 \mathrm{~W}$ and the slope efficiency $67 \%$. The laser power-conversion efficiency was $47.6 \%$ close to the quantum limit.

\section{References}

[1] D. Y. Shen, J. K. Sahu, W. A. Clarkson, "Highly efficient Erbium-Ytterbium co-doped fiber laser with 188W output power at $1565 \mathrm{~nm}$ ", in CLEO/Europe-EQEC 2005 Munich 12-17 Jun 2005, paper CJ1

[2] Y. Jeong, J. K. Sahu, D. B. Soh, C. Codemard, J. Nilsson, "Tunable single-frequency ytterbium-sensitized erbium-doped fiber MOPA source with $150 \mathrm{~W}(51.8 \mathrm{dBm})$ of output power at $1563 \mathrm{~nm}$ ", in OFC 2005 Anaheim 6-11 Mar 2005 paper PDP1 (Postdeadline)

[3] Y. Jeong, P. Dupriez, J. K. Sahu, J. Nilsson, D. Shen, W. A. Clarkson, S. D. Jackson, "Thulium-ytterbium co-doped fiber laser with 75W of output power at 2 microns," SPIE European Symposium on Optics and Photonics for Defence \& Security London 25-28 Oct 2004 Paper.5620-4

[4] K. S. Chiang, "Stimulated Raman scattering in a multimode optical fiber evolution of modes in Stokes waves", Opt. Lett. 17, 352 (1992)

[5] S. H. Baek and W. B. Roh, "Single-mode Raman fiber laser based on a multimode fiber", Opt. Lett. 29, 153-155 (2004)

[6] J. N. Jang, Y. Jeong, J. K. Sahu, M. Ibsen, C. A. Codemard, R. Selvas, D. C. Hanna and J. Nilsson, "Cladding-pumped continuous-wave Raman fiber laser", in Conference on Lasers and Electro-Optics (CLEO 2003),Vol. 89 of OSA Trends in Optics and Photonics Series (Optical Society of America, Washington, D.C., 2003), paper CWL-1

[7] C. S. Goh, M. R. Mokhtar, S. A. Butler, S. Y. Set, K. Kikuchi, M. Ibsen, "Wavelength tuning of fiber Bragg gratings over 90nm using a simple tuning package", IEEE Photonics Technology Letters 15, 557-559 (2003)

[8] J.-C. Bouteiller, "Spectral Modeling of Raman fiber lasers", IEEE Photon. Technol, Lett. 15, 1698-1700 (2003) 